\title{
Singularity-Free Dynamic Equations of Spacecraft-Manipulator Systems
}

\author{
Pål J. From* Kristin Y. Pettersen* Jan T. Gravdahl* \\ * Engineering Cybernetics, Norwegian University of Science $\&$ \\ Technology, Norway
}

\begin{abstract}
In this paper we derive the singularity-free dynamic equations of spacecraftmanipulator systems using a minimal representation. Spacecraft are normally modeled using Euler angles, which leads to singularities, or Euler parameters, which is not a minimal representation and thus not suited for Lagrange's equations. We circumvent these issues by introducing quasi-coordinates which allows us to derive the dynamics using minimal and globally valid non-Euclidean configuration coordinates. This is a great advantage as the configuration space of a spacecraft is non-Euclidean. We thus obtain a computationally efficient and singularity-free formulation of the dynamic equations with the same complexity as the conventional Lagrangian approach. The closed form formulation makes the proposed approach well suited for system analysis and model-based control. This paper focuses on the dynamic properties of free-floating and free-flying spacecraft-manipulator systems and we show how to calculate the inertia and Coriolis matrices in such a way that this can be implemented for simulation and control purposes without extensive knowledge of the mathematical background.
\end{abstract}

Keywords: Spacecraft-manipulator dynamics, robot modeling, singularities, quasi-coordinates.

\section{INTRODUCTION}

A good understanding of the dynamics of spacecraftmanipulator systems is important as these systems are emerging as an alternative to human operation in space (Hughes, 2002; Moosavian and Papadopoulos, 2007). Operations include assembling, repair, refuelling, maintenance and operations of satellites and space stations. Due to the enormous risks and costs involved with launching humans into space, robotic solutions evolve as the most cost-efficient and reliable solution.

Modeling spacecraft-manipulator systems is quite different from standard robot modeling. Firstly, the manipulator is mounted on a free-floating (unactuated) or free-flying (actuated) spacecraft. There is thus no obvious way to choose the inertial frame. Secondly, the motion of the manipulator affects the motion of the base, which results in a set of dynamic equations different from the fixedbase case due to the dynamic coupling. Finally, we need to consider the effects of the free fall environment.

Robustness of these systems is still a major concern for space operators. We therefore derive the singularity-free dynamic equations of spacecraft-manipulator systems using a Lagrangian framework. It is a well known fact that the kinematics of a rigid body contains singularities if the Euler angles are used to represent the orientation and the joint topology is not taken into account. One solution to this problem is to use a non-minimal representation such as the unit quaternion. These are, however, not generalized coordinates and can thus not be used in Lagrange's equations. This is a major drawback when it comes to modeling vehicle-manipulator systems as most methods used for robot modeling are based on the Lagrangian approach.
It is thus a great advantage if also the vehicle dynamics can be derived from the Lagrange equations.

The use of Lie groups and algebras as a mathematical basis for the derivation of the dynamics of multibody systems can be used to overcome this problem (Selig, 2000). We then choose the coordinates generated by the Lie algebra as local Euclidean coordinates which allows us to describe the dynamics locally. For this approach to be valid globally the total configuration space needs to be covered by an atlas of local exponential coordinate patches. The appropriate equations must then be chosen for the current configuration. The geometric approach presented in Bullo and Lewis (2004) can then be used to obtain a globally valid set of dynamic equations on a single Lie group, such as a spacecraft with no robot attached.

Even though combinations of Lie groups can be used to represent multibody systems, the formulation is very complex and not suited for implementation in a simulation environment. In Kwatny and Blankenship (2000) quasicoordinates was used to derive the dynamic equations of fixed-base robotic manipulators using Poincaré's formulation of the Lagrange equations. In Kozlowski and Herman (2008) several control laws using a quasi-coordinate approach were presented, but only robots with conventional 1-DoF joints were considered. Common for all these methods is, however, that the configuration space of the system is described as $q \in \mathbb{R}^{n}$. This is not a problem when dealing with $1-\mathrm{DoF}$ revolute or prismatic joints but more complicated joints such as ball-joints or free-floating joints then need to be modeled as compound kinematic joints (Kwatny and Blankenship, 2000), i.e., a combination of 1-DoF simple kinematic joints. For joints that use the 


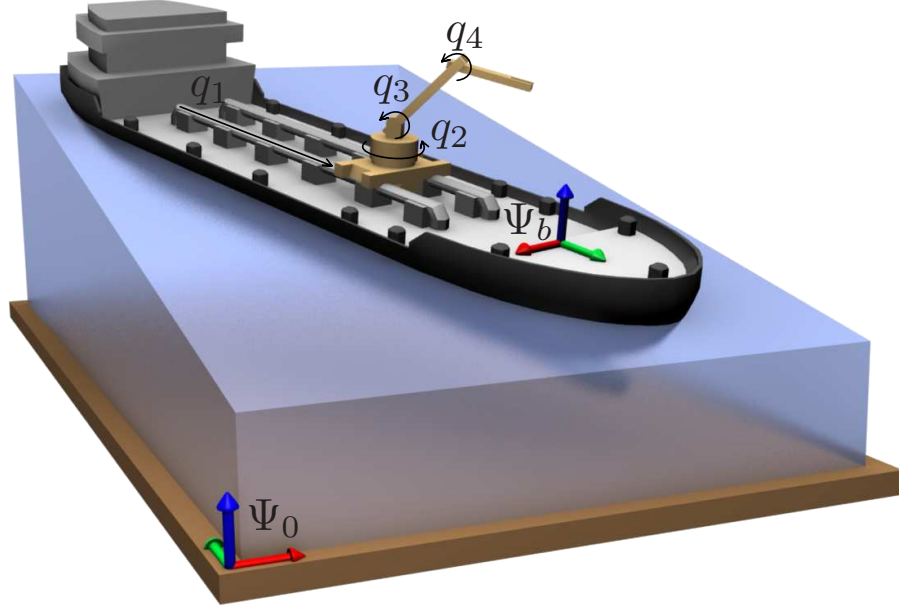

Fig. 1. Model setup for a robot attached to a vehicle with coordinate frame $\Psi_{b}$ and inertial reference frame $\Psi_{0}$.

Euler angles to represent the orientation this leads to singularities in the representation.

In this paper we follow the generalized Lagrangian approach presented in Duindam and Stramigioli (2008) which allows us to combine the Euclidean joints and more general joints, i.e., joints that can be described by the Lie group $S E(3)$ or one of its ten subgroups, and we extend these ideas to spacecraft-manipulator systems. There are several advantages in following this approach. The use of quasi-coordinates, i.e., velocity coordinates that are not simply the time derivative of the position coordinates, allows us to include joints (or transformations) with a different topology than that of $\mathbb{R}^{n}$. For example, for a spacecraft we can represent the transformation from the inertial frame to the spacecraft body frame as a freefloating joint with configuration space $S E(3)$ and we avoid the singularity-prone kinematic relations between the inertial frame and the body frame velocities that normally arise in deriving the spacecraft dynamics (Hughes, 2002).

This approach differs from previous work in that it allows us to derive the dynamic equations of vehicle-manipulator systems for vehicles with a configuration space different from $\mathbb{R}^{n}$. The dynamics are expressed (locally) in exponential coordinates $\phi$, but the final equations are evaluated at $\phi=0$. This has two main advantages. Firstly, the dynamics do not depend on the local coordinates as these are eliminated from the equations and the global position and velocity coordinates are the only state variables. This makes the equations valid globally. Secondly, evaluating the equations at $\phi=0$ greatly simplifies the dynamics and make the equations suited for implementation in simulation software. We also note that the approach is well suited for model-based control as the equations are explicit and without constraints. The fact that the configuration space of the spacecraft is a Lie group also simplifies the implementation. Even though the expressions in the derivation of the dynamics are somewhat complex, we have several tools from the Lie theory that allows us to write the final expressions in a very simple form.

The paper is organized as follows. Section 2 gives the detailed mathematical background for the proposed approach. This section can be skipped and practitioners mainly interested in implementation can go straight to Section 4. Section 3 presents the state of the art in spacecraft-manipulator modeling and in Section 4 the dynamic equations for spacecraft-manipulator systems and the effects of the free-floating base are treated in detail. To the authors best knowledge spacecraft-manipulator systems have not been studied in detail in literature using the framework presented here. The matrix representation of the dynamics and how to implement this is presented in great detail which allows the readers to implement this in a simulation or control environment without having to perform all the detailed computations themselves.

\section{DYNAMIC EQUATIONS OF VEHICLE-MANIPULATOR SYSTEMS}

We extend the classical dynamic equations for a serial manipulator arm with 1-DoF joints to include the motion of the spacecraft.

\subsection{Vehicle-Manipulator Kinematics}

Consider the setup of Figure 1 describing a general $n$-link robot manipulator arm attached to a vehicle. Choose an inertial coordinate frame $\Psi_{0}$, a frame $\Psi_{b}$ rigidly attached to the vehicle, and $n$ frames $\Psi_{i}$ (not shown) attached to each link $i$ at the center of mass with axes aligned with the principal directions of inertia. Finally, choose a vector $q \in \mathbb{R}^{n}$ that describes the configuration of the $n$ joints. Using standard notation (Murray et al., 1994), we describe the pose of each frame $\Psi_{i}$ relative to $\Psi_{0}$ as a homogeneous transformation matrix $g_{0 i} \in S E(3)$ of the form

$$
g_{0 i}=\left[\begin{array}{cc}
R_{0 i} & p_{0 i} \\
0 & 1
\end{array}\right] \in \mathbb{R}^{4 \times 4}
$$

with rotation matrix $R_{0 i} \in S O(3)$ and translation vector $p_{0 i} \in \mathbb{R}^{3}$. This pose can also be described using the vector of joint coordinates $q$ as

$$
g_{0 i}=g_{0 b} g_{b i}=g_{0 b} g_{b i}(q) .
$$

The vehicle pose $g_{0 b}$ and the joint positions $q$ thus fully determine the configuration state of the robot. We use $g_{0 b}(6 \mathrm{DoF})$ to represent the vehicle configuration, but the actual configuration space of the vehicle may be a subspace of $S E(3)$ of dimension $m<6$. In our case, a spacecraft has configuration space $S O(3)$ with dimension $m=3$. In the case of $m<6$ we define a selection matrix $H \in \mathbb{R}^{6 \times m}$ such that the twist is given by

$$
V_{0 b}^{b}=H \tilde{V}_{0 b}^{b}
$$

where $\tilde{V}_{0 b}^{b} \in \mathbb{R}^{m}$ determines the $m$-dimensional velocity state of the vehicle. The spatial velocity of each link can be expressed using twists:

$$
V_{0 i}^{0}=\left[\begin{array}{c}
v_{0 i}^{0} \\
\omega_{0 i}^{0}
\end{array}\right]=V_{0 b}^{0}+V_{b i}^{0}=\operatorname{Ad}_{g_{0 b}}\left(H \tilde{V}_{0 b}^{b}+J_{i}(q) \dot{q}\right)
$$

where $v_{0 i}^{0}$ and $\omega_{0 i}^{0}$ are the linear and angular velocities, respectively, of link $i$ relative to the inertial frame, $J_{i}(q) \in$ $\mathbb{R}^{6 \times n}$ is the geometric Jacobian of link $i$ relative to $\Psi_{b}$, the adjoint is defined as $\operatorname{Ad}_{g}:=\left[\begin{array}{cc}R & \hat{p} R \\ 0 & R\end{array}\right] \in \mathbb{R}^{6 \times 6}$, and $\hat{p} \in \mathbb{R}^{3 \times 3}$ is the skew symmetric matrix such that $\hat{p} x=p \times x$ for all $p, x \in \mathbb{R}^{3}$. The velocity state is thus fully determined given the twist $V_{0 b}^{b}$ of the vehicle and the joint velocities $\dot{q}$. 


\subsection{Vehicle-Manipulator Dynamics}

The previous section shows how the kinematics of the system can be described in terms of the (global) state variables $g_{0 b}, q, V_{0 b}^{b}$, and $\dot{q}$. To derive the dynamics of the complete mechanism (including the $m$-DoF between $\Psi_{0}$ and $\left.\Psi_{b}\right)$ in terms of these state variables, we follow the generalized Lagrangian method introduced by Duindam and Stramigioli (2008). This method gives the dynamic equations for a general mechanism described by a set $Q=\left\{Q_{i}\right\}$ of configuration states $Q_{i}$ (not necessarily Euclidean), a vector $v$ of velocity states $v_{i} \in \mathbb{R}^{n_{i}}$, and several mappings that describe the local Euclidean structure of the configuration states and their relation to the velocity states. More precisely, the neighborhood of every state $\vec{Q}_{i}$ is locally described by a set of Euclidean coordinates $\phi_{i} \in$ $\mathbb{R}^{n_{i}}$ as $Q_{i}=\Phi_{i}\left(\bar{Q}_{i}, \phi_{i}\right)$ with $\Phi_{i}\left(\bar{Q}_{i}, 0\right)=\bar{Q}_{i} . \Phi_{i}\left(\bar{Q}_{i}, \phi_{i}\right)$ defines a local diffeomorphism between a neighborhood of $0 \in \mathbb{R}^{n_{i}}$ and a neighborhood of $\bar{Q}_{i}$.

We start by deriving an expression for the kinetic coenergy of a mechanism, expressed in coordinates $Q, v$, but locally parameterized by the coordinate mappings for each joint. For joints that can be described by a matrix Lie group, this mapping can be given by the exponential map (Murray et al., 1994). Let $\phi \in \operatorname{se}(n, \mathbb{R})$ be the Lie algebra of $S E(3)$, then the exponential map $\exp (\phi)$ is given by

$$
e^{\hat{\phi}}=I+\hat{\phi}+\frac{\hat{\phi}^{2}}{2} \cdots=\sum_{n=0}^{\infty} \frac{\hat{\phi}^{n}}{n !}
$$

where $I$ (no subscript) is the identity matrix. The dynamics are thus expressed in local coordinates $\phi$ for configuration and $v$ for velocity, and we consider $Q$ a parameter. After taking partial derivatives of the Lagrangian function, we evaluate the results at $\phi=0$ (i.e. at configuration $Q$ ) to obtain the dynamics expressed in global coordinates $Q$ and $v$ as desired. We note that even though local coordinates $\phi$ appear in the derivations of the various equations, the final equations are all evaluated at $\phi=0$ and hence these final equations do not depend on local coordinates. The global coordinates $Q$ and $v$ are the only dynamic state variables and the equations are valid globally, without the need for coordinate transitions between various areas of the configuration space. Note also that taking the partial derivatives of the Lagrangian and evaluating at $\phi=0$ greatly simplifies (5) and the closed form expressions of the exponential map is not needed. This fact greatly simplifies the final equations.

In general, the topology of a Lie group is not Euclidean. When deriving the dynamic equations for spacecraft from the Lagrangian, this is normally dealt with by introducing a transformation matrix that relates the local and global velocity variables. However, forcing the dynamics into a vector representation in this way, without taking the topology of the configuration space into account, leads to singularities in the representation or other deficiencies. To preserve the topology of the configuration space we will use quasi-coordinates, i.e. velocity coordinates that are not simply the time-derivative of position coordinates, but given by a linear relation. Thus, there exist differentiable matrices $S_{i}$ such that we can write $v_{i}=S_{i}\left(Q_{i}, \phi_{i}\right) \dot{\phi}_{i}$ for every $Q_{i}$. For Euclidean joints this relation is given by the identity map while for joints with a Lie group topology we can use the exponential map to derive this relation.
Given a mechanism with coordinates formulated in this generalized form, we can write its kinetic energy as $\mathcal{T}(Q, v)=\frac{1}{2} v^{\top} M(Q) v$ with $M(Q)$ the inertia matrix in coordinates $Q$ and $v$ the stacked velocities of the vehicle and the robot joints. The dynamics then satisfy

$$
M(Q) \dot{v}+C(Q, v) v=\tau
$$

with $\tau$ the vector of external and control wrenches (collocated with $v)$, and $C(Q, v)$ the matrix describing Coriolis and centrifugal forces given by

$$
\begin{array}{r}
C_{i j}(Q, v):=\left.\sum_{k, l}\left(\frac{\partial M_{i j}}{\partial \phi_{k}} S_{k l}^{-1}-\frac{1}{2} S_{k i}^{-1} \frac{\partial M_{j l}}{\partial \phi_{k}}\right)\right|_{\phi=0} v_{l} \\
+\left.\sum_{k, l, m, s}\left(S_{m i}^{-1}\left(\frac{\partial S_{m j}}{\partial \phi_{s}}-\frac{\partial S_{m s}}{\partial \phi_{j}}\right) S_{s k}^{-1} M_{k l}\right)\right|_{\phi=0} v_{l}
\end{array}
$$

We refer to Duindam and Stramigioli (2008) for details. To apply this general result to systems of the form of Figure 1, we write $Q=\left\{g_{0 b}, q\right\}$ as the set of configuration states where $g_{0 b}$ is the Lie group $S O(3)$, and $v=\left[\left(V_{0 b}^{b}\right)^{\top} \dot{q}^{\top}\right]^{\top}$ as the vector of velocity states. The local Euclidean structure for the state $g_{0 b}$ is given by exponential coordinates, while the state $q$ is itself globally Euclidean. Mathematically, we can express configurations $\left(g_{0 b}, q\right)$ around a fixed state $\left(\bar{g}_{0 b}, \bar{q}\right)$ as

$g_{0 b}=\bar{g}_{0 b} \exp \left(\sum_{j=1}^{6} b_{j}\left(\phi_{b}\right)_{j}\right), \quad q_{i}=\bar{q}_{i}+\phi_{i} \forall i \in\{1 \ldots n\}$

with $b_{j}$ the standard basis elements of the Lie algebra so(3). From expression (4) for the twist of each link in the mechanism, we can derive an expression for the total kinetic energy. Let $I_{b} \in \mathbb{R}^{6 \times 6}$ and $I_{i} \in \mathbb{R}^{6 \times 6}$ denote the constant positive-definite diagonal inertia tensor of the base and link $i$ (expressed in $\Psi_{i}$ ), respectively. The kinetic energy $\mathcal{T}_{i}$ of link $i$ then follows as

$$
\begin{aligned}
& \mathcal{T}_{i}=\frac{1}{2}\left(V_{0 i}^{i}\right)^{\top} I_{i} V_{0 i}^{i} \\
& =\frac{1}{2}\left(\left(\tilde{V}_{0 b}^{b}\right)^{\top} H^{\top}+\dot{q}^{\top} J_{i}(q)^{\top}\right) \operatorname{Ad}_{g_{i b}}^{\top} I_{i} \operatorname{Ad}_{g_{i b}}\left(H \tilde{V}_{0 b}^{b}+J_{i}(q) \dot{q}\right) \\
& =\frac{1}{2}\left[\left(\tilde{V}_{0 b}^{b}\right)^{\top} \dot{q}^{\top}\right] M_{i}(q)\left[\begin{array}{c}
\tilde{V}_{0 b}^{b} \\
\dot{q}
\end{array}\right]=\frac{1}{2} v^{\top} M_{i}(q) v
\end{aligned}
$$

with $M_{b}=\left[\begin{array}{cc}I_{b} & 0 \\ 0 & 0\end{array}\right] \in \mathbb{R}^{(m+n) \times(m+n)}$ for the vehicle and

$$
M_{i}(q):=\left[\begin{array}{cccc}
H^{\top} \operatorname{Ad}_{q_{i b}}^{\top} I_{i} \operatorname{Ad}_{g_{i b}} H & H^{\top} \operatorname{Ad}_{q_{i b}}^{\top} I_{i} \operatorname{Ad}_{g_{i b}} J_{i} \\
J_{i}^{\top} \operatorname{Ad}_{g_{i b}}^{\top} I_{i} \operatorname{Ad}_{g_{i b}} H & J_{i}^{\top} \operatorname{Ad}_{g_{i b}}^{\top} I_{i} \operatorname{Ad}_{g_{i b}} J_{i}
\end{array}\right]
$$

for the links. The total kinetic energy of the mechanism is given by the sum of the kinetic energies of the mechanism links and the vehicle, that is,

$$
\mathcal{T}(q, v)=\frac{1}{2} v^{\top} \underbrace{\left(\left[\begin{array}{cc}
I_{b} & 0 \\
0 & 0
\end{array}\right]+\sum_{i=1}^{n} M_{i}(q)\right)}_{\text {inertia matrix } M(q)} v
$$

with $M(q)$ the inertia matrix of the total system. Note that neither $\mathcal{T}(q, v)$ nor $M(q)$ depend on the pose $g_{0 b}$ nor the choice of inertial reference frame $\Psi_{0}$. We can now write (6) in block-form as follows

$$
\left[\begin{array}{cc}
M_{V V} & M_{q V}^{\top} \\
M_{q V} & M_{q q}^{\top}
\end{array}\right]\left[\begin{array}{c}
\tilde{\tilde{V}}_{0 b}^{b} \\
\ddot{q}
\end{array}\right]+\left[\begin{array}{cc}
C_{V V} & C_{V q} \\
C_{q V} & C_{q q}
\end{array}\right]\left[\begin{array}{c}
\tilde{V}_{0 b}^{b} \\
\dot{q}
\end{array}\right]=\left[\begin{array}{c}
\tau_{V} \\
\tau_{q}
\end{array}\right]
$$

Here the subscript $V$ refers to the first $m$ entries and $q$ the remaining $n-m$ entries. 


\subsection{Vehicles with Configurations Space SE(3)}

The configuration space of a free-floating vehicle, such as a spacecraft, can be described by the matrix Lie group $S E(3)$. In this case we have the mapping (Duindam, 2006)

$$
V_{0 b}^{b}=\left(I-\frac{1}{2} \operatorname{ad}_{\phi_{V}}+\frac{1}{6} \operatorname{ad}_{\phi_{V}}^{2}-\ldots\right) \dot{\phi}_{V}
$$

with $\operatorname{ad}_{p}=\left[\begin{array}{cc}\hat{p}_{4} \ldots 6 & \hat{p}_{1 \ldots 3} \\ 0 & \hat{p}_{4} \ldots 6\end{array}\right] \in \mathbb{R}^{6 \times 6}$ for $p \in \mathbb{R}^{6}$ relating the local and global velocity variables. The corresponding matrices $S_{i}$ can be collected in one block-diagonal matrix $S \in \mathbb{R}^{(6+n) \times(6+n)}$ given by

$$
S(Q, \phi)=\left[\begin{array}{cc}
\left(I-\frac{1}{2} \operatorname{ad}_{\phi_{V}}+\frac{1}{6} \operatorname{ad}_{\phi_{V}}^{2}-\ldots\right) & 0 \\
0 & I
\end{array}\right] .
$$

We note that when differentiating with respect to $\phi$ and substituting $\phi=0$ this simplifies the expression substantially.

To compute the matrix $C(Q, v)$ for our system, we can use the observations that $M(q)$ is independent of $g_{0 b}$, that $S(Q, \phi)$ is independent of $q$, and that $S(Q, 0) \equiv I$. Furthermore, the partial derivative of $M$ with respect to $\phi_{V}$ is zero since $M$ is independent of $g_{0 b}$, and the second term of (7) is only non-zero for the $C_{V V}$ block of $C(Q, v)$. Firstly, $C_{V V}$ depends on both the first and the second term in (7). We have $i, j=1 \ldots 6$. Note that $\frac{\partial M_{i j}}{\partial \phi_{k}}=0$ for $k<7$ and $\frac{\partial S_{i j}}{\partial \phi_{k}}=0$ for $i, j, k>6$. This simplifies $C_{V V}$ to

$$
\begin{aligned}
C_{i j}(Q, v)=\sum_{k=7}^{6+n}( & \frac{\partial M_{i j}}{\partial \phi_{k}}-\underbrace{\frac{1}{2} \frac{\partial M_{j k}}{\partial \phi_{i}}}_{=0})\left.\right|_{\phi=0} v_{k} \\
& +\left.\sum_{k=1}^{6}\left(\frac{\partial S_{i j}}{\partial \phi_{k}}-\frac{\partial S_{i k}}{\partial \phi_{j}}\right)\right|_{\phi=0}(M(q) v)_{k} .
\end{aligned}
$$

Furthermore, if we write $S=\left(I-\frac{1}{2} \operatorname{ad}_{\phi_{V}}+\frac{1}{6} \operatorname{ad}_{\phi_{V}}^{2}-\ldots\right)$ we note that after differentiating and evaluating at $\phi=0$, $\sum \frac{\partial S_{i j}}{\partial \phi_{k}}$ is equal to $-\frac{1}{2} \operatorname{ad}_{e_{k}}$ where $e_{k}$ is a 6 -vector with 1 in the $k^{\text {th }}$ entry and zeros elsewhere. Similarly, $\sum \frac{\partial S_{i k}}{\partial \phi_{j}}$ is equal to $\frac{1}{2} \operatorname{ad}_{e_{k}}$. This is then multiplied by the $k^{\text {th }}$ element of $M(q) v$ when differentiating with respect to $\phi_{k}$ so that

$$
C_{V V}(Q, v)=\sum_{k=1}^{6} \frac{\partial M_{V V}}{\partial q_{k}} \dot{q}_{k}-\operatorname{ad}_{(M(q) v)_{V}}
$$

where $(M(q) v)_{V}$ is the vector of the first 6 entries (corresponding to $\left.V_{0 b}^{b}\right)$ of the vector $M(q) v$.

$C_{V q}(Q, v)$, i.e., $i=1 \ldots 6$ and $j=7 \ldots(6+n)$, is found in a similar manner. First we note that $\frac{\partial M_{j k}}{\partial \phi_{i}}=0$ for $i=1 \ldots 6$ and that $\frac{\partial S_{i j}}{\partial \phi_{k}}=0$ and $\frac{\partial S_{i k}}{\partial \phi_{j}}=0$ for $j=7 \ldots(6+n)$, so only the first part is non-zero and we get

$$
C_{V q}(Q, v)=\sum_{k=1}^{6} \frac{\partial M_{V q}}{\partial q_{k}} \dot{q}_{k}
$$

Finally, the terms $C_{q V}$ and $C_{q q}$ depend only on the first part of (7) and can be written as (From et al., 2009)

$$
\begin{aligned}
C_{q V} & =\sum_{k=1}^{n} \frac{\partial M_{q V}}{\partial q_{k}} \dot{q}_{k}-\frac{1}{2} \frac{\partial^{\top}}{\partial q}\left(\left[\begin{array}{ll}
M_{V V} & M_{q V}^{\top}
\end{array}\right]\left[\begin{array}{c}
V_{0 b}^{b} \\
\dot{q}
\end{array}\right]\right), \\
C_{q q} & =\sum_{k=1}^{n} \frac{\partial M_{q q}}{\partial q_{k}} \dot{q}_{k}-\frac{1}{2} \frac{\partial^{\top}}{\partial q}\left(\left[\begin{array}{ll}
M_{q V} & M_{q q}^{\top}
\end{array}\right]\left[\begin{array}{c}
V_{0 b}^{b} \\
\dot{q}
\end{array}\right]\right) .
\end{aligned}
$$

The $C$-matrix is thus given by

$$
\begin{aligned}
& C(Q, v)=\sum_{k=1}^{n} \frac{\partial M}{\partial q_{k}} \dot{q}_{k} \\
& -\frac{1}{2}\left[\frac{\partial^{\top}}{\partial q}\left(\left[\begin{array}{cc}
2 \operatorname{ad}_{V V} & M_{q V}^{\top}
\end{array}\right]\left[\begin{array}{c}
V_{0 b}^{b} \\
\dot{q}
\end{array}\right]\right) \frac{\partial^{\top}}{\partial q}\left(\left[\begin{array}{cc}
M_{q V} & M_{q q}^{\top}
\end{array}\right]\left[\begin{array}{c}
V_{0 b}^{b} \\
\dot{q}
\end{array}\right]\right)\right] .
\end{aligned}
$$

\subsection{Vehicles with Configurations Space SO(3)}

The dynamics of a vehicle-manipulator system for a vehicle with configuration space $S O(3)$ are derived in the same way. The velocity state is thus fully determined by only three variables and we choose $H$ so that

$$
V_{0 b}^{b}=H \tilde{V}_{0 b}^{b}, \quad H=\left[\begin{array}{ll}
0_{3 \times 3} & I_{3 \times 3}
\end{array}\right]^{\top} .
$$

The corresponding matrices $S_{i} \in \mathbb{R}^{(3+n) \times(3+n)}$ can be collected in one block-diagonal matrix $S$ given by

$$
S(Q, \phi)=\left[\begin{array}{cc}
\left(I-\frac{1}{2} \hat{\phi}_{V}+\frac{1}{6} \hat{\phi}_{V}^{2}+\ldots\right) & 0 \\
0 & I
\end{array}\right] .
$$

The precise computational details of the partial derivatives follow the same steps as for the $S E(3)$ case except for $C_{V V}$. Note that $\frac{\partial M_{i j}}{\partial \phi_{k}}=0$ for $k<4$ and $\frac{\partial S_{i j}}{\partial \phi_{k}}=0$ for $i, j, k>3$. When differentiating and evaluating at $\phi=0$ the matrices $\sum \frac{\partial S_{i j}}{\partial \phi_{k}}$ are equal to $-\frac{1}{2} \hat{e}_{k}$ where $e_{k}$ is a 3 -vector with 1 in the $k^{\text {th }}$ entry and zeros elsewhere. Similarly, $\sum \frac{\partial S_{i k}}{\partial \phi_{j}}$ is equal to $\frac{1}{2} \hat{e}_{k}$. We then get

$$
C_{V V}(Q, v)=\sum_{k=1}^{6} \frac{\partial M_{V V}}{\partial q_{k}} \dot{q}_{k}-(\widehat{M(q) v})_{\tilde{V}}
$$

where $(M(q) v)_{\tilde{V}}$ is the vector of the first three entries of the vector $M(q) v$ (corresponding to $\tilde{V}_{0 b}^{b}$ ).

\section{STATE OF THE ART SPACECRAFT-MANIPULATOR DYNAMICS}

\subsection{State of the Art Spacecraft Dynamics}

The attitude of a spacecraft is normally described by the Euler parameters, or unit quaternion. This is motivated by their properties as a nonsingular representation. We note that this is not the minimal representation, nor generalized coordinates, and thus not suited for the Lagrangian approach. Also, when transforming back to Euler angles from the unit quaternion representation a singularity is present for $\theta= \pm \frac{\pi}{2}$. Any positive rotation $\psi$ about a fixed unit vector $n$ can be represented by the four-tuple $Q=\left[\eta \epsilon^{\top}\right]^{\top}$, where $\eta=\cos \left(\frac{\psi}{2}\right) \in \mathbb{R}$ is the scalar part and $\epsilon=\sin \left(\frac{\psi}{2}\right) n \in \mathbb{R}^{3}$ the vector part. The kinematic differential equations can now be given by (Hughes, 2002)

$$
\dot{\eta}=-\frac{1}{2} \epsilon^{\top} \omega_{0 b}^{0}, \quad \dot{\epsilon}=\frac{1}{2}\left(\eta I_{b}+\hat{\epsilon}\right) \omega_{0 b}^{0}
$$


where $\omega_{0 b}^{0}$ is the angular velocity of the body frame with respect to the orbit frame and $I_{b}$ the spacecraft inertia matrix. The attitude dynamics are given by

$$
I_{b} \dot{\omega}_{0 b}^{0}+\hat{\omega}_{0 b}^{0} I_{b} \omega_{0 b}^{0}=\tau \text {. }
$$

\subsection{State of the Art Spacecraft-Manipulator Dynamics}

The equations of motion of a spacecraft-manipulator system can be written as

$$
M(Q) \dot{v}+C(Q, v) v=\tau .
$$

Here, $v=\left[\begin{array}{ll}\dot{r}_{0}^{\top} & \left(\omega_{0 b}^{0}\right)^{\top} \dot{q}^{\top}\end{array}\right]^{\top}$ where $r_{0}$ is the position of the center of mass of the spacecraft, $\omega_{0 b}^{0}$ the angular velocity of the spacecraft and $q$ is the joint position of the manipulator.

Alternatively we can use the center of mass of the whole system to represent the translational motion. Then $v=$ $\left[\dot{r}_{c m}^{\top}\left(\omega_{0 b}^{0}\right)^{\top} \dot{q}^{\top}\right]^{\top}$ where $\dot{r}_{c m}$ is the linear velocity of the center of mass of the spacecraft-manipulator system. This is decoupled from the angular velocity $\omega_{0 b}^{0}$ and the inertia matrix for a free-flying spacecraft-manipulator system can be written as (Dubowsky and Papadopoulos, 1993)

$$
M=\left[\begin{array}{ccc}
m I & 0 & 0 \\
0 & M_{\omega \omega} & M_{q \omega}^{\top} \\
0 & M_{q \omega} & M_{q q}
\end{array}\right]
$$

where $m$ is the total mass of the system. The Euler angle rates $\dot{\Theta}_{0 b}$ relate to $\omega_{0 b}^{0}$ by

$$
\dot{\Theta}_{0 b}=T_{\Theta_{0 b}}\left(\Theta_{0 b}\right) \omega_{0 b}^{0} .
$$

Again $T_{\Theta_{0 b}}\left(\Theta_{0 b}\right)$ is singular at isolated points. The control torques are given by $\tau=\left[\begin{array}{lll}\tau_{v}^{\top} & \tau_{\omega}^{\top} & \tau_{q}^{\top}\end{array}\right]^{\top}$ where $\tau_{v}$ is the spacecraft forces, $\tau_{\omega}$ is the spacecraft moments, and $\tau_{q}$ is the manipulator torques.

Other models are also available depending on the actuators available to control the spacecraft. In the case where $\tau_{v}, \tau_{w} \neq 0$ (free-flying space robots) the center of mass of the system is not constant, but described by the variable

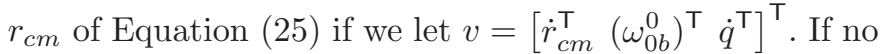
external forces act on the system and the spacecraft is not actuated, the center of mass does not accelerate, i.e., the system linear momentum is constant and $\dot{r}_{c m}=0$. This can be used to simplify the equations to an $n$-dimensional system with inertia matrix $M_{r}=M_{q q}-M_{q \omega} M_{\omega \omega}^{-1} M_{q \omega}^{\top}$ and we get the reduced system by eliminating $\omega$

$$
M_{r}(Q) \ddot{q}+C_{r}(Q, v) \dot{q}=\tau_{q} .
$$

The attitude of the spacecraft is then found from

$$
\omega=-M_{\omega \omega}^{-1} M_{q \omega}^{\top} \dot{q} \text {. }
$$

\section{THE PROPOSED APPROACH}

\subsection{Configuration space $S E(3)$}

The configuration space of a spacecraft can be described by the Lie group $S E(3)$ with respect to an orbit-fixed frame. The dynamic equations can be written as

$$
\left[\begin{array}{cc}
M_{V V} & M_{q V}^{\top} \\
M_{q V} & M_{q q}
\end{array}\right]\left[\begin{array}{c}
\dot{V}_{0 b}^{b} \\
\ddot{q}
\end{array}\right]+\left[\begin{array}{cc}
C_{V V} & C_{V q} \\
C_{q V} & C_{q q}
\end{array}\right]\left[\begin{array}{c}
V_{0 b}^{b} \\
\dot{q}
\end{array}\right]=\left[\begin{array}{c}
\tau_{V} \\
\tau_{q}
\end{array}\right]
$$

$C(Q, v)=\sum_{k=1}^{n} \frac{\partial M}{\partial q_{k}} \dot{q}_{k}$

$-\frac{1}{2}\left[\frac{\partial^{\top}}{\partial q}\left(\left[\begin{array}{c}2 \operatorname{ad}_{V V} M_{q V}^{\top} \\ \left.M^{\top}\right)_{V}\end{array}\left[\begin{array}{c}V_{0 b}^{b} \\ \dot{q}\end{array}\right]\right) \frac{\partial^{\top}}{\partial q}\left(\left[\begin{array}{cc}M_{q V} & M_{q q}^{\top}\end{array}\right]\left[\begin{array}{c}V_{0 b}^{b} \\ \dot{q}\end{array}\right]\right)\right]\right.$.

The dynamics are singularity-free and with state variables $Q=\left\{g_{0 b} \in S E(3), q \in \mathbb{R}^{n}\right\}$ and $v=\left[\left(V_{0 b}^{b}\right)^{\top} \dot{q}^{\top}\right]^{\top} \in \mathbb{R}^{6+n}$, and valid for both for actuated and unactuated spacecraft.

\subsection{Configuration space $S O(3)$}

For free-floating spacecraft we have $\tau_{V}=0$ and $r_{c m}$ is thus decoupled from the angular velocity of the spacecraft so we write the kinetic energy of link $i$ of the system as

$$
\mathcal{T}_{i}=\frac{1}{2}\left[\left(\omega_{0 b}^{0}\right)^{\top} \dot{q}^{\top}\right] M_{i}(q)\left[\begin{array}{c}
\omega_{0 b}^{0} \\
\dot{q}
\end{array}\right]=\frac{1}{2} v^{\top} M_{i}(q) v
$$

where

$$
M_{i}(q):=\left[\begin{array}{cccccc}
H^{\top} \operatorname{Ad}_{q_{i b}}^{\top} I_{i} \operatorname{Ad}_{g_{i b}} H & H^{\top} \operatorname{Ad}_{q_{i b}}^{\top} I_{i} \operatorname{Ad}_{g_{i b}} J_{i} \\
J_{i}^{\top} \operatorname{Ad}_{g_{i b}}^{\top^{\prime}} I_{i} \operatorname{Ad}_{g_{i b}} H & J_{i}^{\top} \operatorname{Ad}_{g_{i b}}^{\top^{i}} I_{i} \operatorname{Ad}_{g_{i b}} J_{i}
\end{array}\right] .
$$

and the inertia matrix is given by substituting this into (10) and $H$ given as in (20). The configuration space is then given by $Q=\left\{R_{0 b}, q\right\}$.

The upper left part of the Coriolis matrix then becomes (following the mathematics of (20-22))

$$
C_{V V}(Q, v)=\sum_{k=1}^{n} \frac{\partial M_{V V}}{\partial q_{k}} \dot{q}_{k}-(\widehat{M(q) v})_{\tilde{V}}
$$

where $(M(q) v)_{\tilde{V}}$ is the vector of the first three entries of the vector $M(q) v$ (corresponding to $\tilde{V}_{0 b}^{b}=\omega_{0 b}^{0}$ ). The dynamic equations can now be written by (30) with velocity state $v=\left[\left(\omega_{0 b}^{0}\right)^{\top} \dot{q}^{\top}\right]^{\top}$ and configuration space $Q=\left\{R_{0 b}, q\right\}$. Again we note that the singularity that normally arises when using the Euler angles is eliminated and the state space $(Q, v)$ is valid globally.

Alternatively we can re-write the mass matrix as

$$
M_{r}=M_{q q}-M_{q V} M_{V V} M_{q V}^{\top} .
$$

The Coriolis matrix is then found by

$$
C_{r}(Q, v)=\sum_{k=1}^{n} \frac{\partial M_{r}}{\partial q_{k}} \dot{q}_{k}-\frac{1}{2} \frac{\partial^{\top}}{\partial q}\left(M_{r} v\right)
$$

with $M_{r}$ as in (34) and the dynamics are described by (28).

Computing the Partial derivatives of $M\left(q_{1}, \ldots, q_{n}\right)$ The partial derivatives of the inertia matrix with respect to $q_{1}, \ldots, q_{n}$ are computed by

$$
\begin{aligned}
& \frac{\partial M\left(q_{1}, \ldots, q_{n}\right)}{\partial q_{k}}= \\
& \sum_{i=k}^{n}\left(\left[\begin{array}{c}
H^{\top} \\
J_{i}^{\top}
\end{array}\right]\left[\frac{\partial^{\top} \operatorname{Ad}_{g_{i b}}}{\partial q_{k}} I_{i} \operatorname{Ad}_{g_{i b}}+\operatorname{Ad}_{g_{i b}}^{\top} I_{i} \frac{\partial \operatorname{Ad}_{g_{i b}}}{\partial q_{k}}\right]\left[\begin{array}{ll}
H & J_{i}
\end{array}\right]\right) \\
& +\sum_{i=k+1}^{n}\left(\left[\frac{\partial^{\top} J_{i}}{\partial q_{k}} \operatorname{Ad}_{g_{i b}}^{0_{m \times m}} I_{i} \operatorname{Ad}_{g_{i b}} H^{\cdots}\right.\right. \\
& \left.\left.\begin{array}{c}
H^{\top} \operatorname{Ad}_{g_{i b}}^{\top} I_{i} \operatorname{Ad}_{g_{i b}} \frac{\partial J_{i}}{\partial q_{k}} \\
\frac{\partial^{\top} J_{i}}{\partial q_{k}} \operatorname{Ad}_{g_{i b}}^{\top} I_{i} \operatorname{Ad}_{g_{i b}} J_{i}+J_{i}^{\top} \operatorname{Ad}_{g_{i b}}^{\top} I_{i} \operatorname{Ad}_{g_{i b}} \frac{\partial J_{i}}{\partial q_{k}}
\end{array}\right]\right) .
\end{aligned}
$$


Proposition 1. Express the velocity of joint $k$ as $V_{(k-1) k}^{(k-1)}=$ $X_{k} \dot{q}_{k}$ for constant $X_{k}$. The partial derivatives of the adjoint matrix is given by

$\frac{\partial \operatorname{Ad}_{g_{i j}}}{\partial q_{k}}=\left\{\begin{aligned} \operatorname{Ad}_{g_{i(k-1)}} \operatorname{ad}_{X_{k}} \operatorname{Ad}_{g_{(k-1) j}} & \text { for } i<k \leq j \\ -\operatorname{Ad}_{g_{i(k-1)}} \operatorname{ad}_{X_{k}} \operatorname{Ad}_{g_{(k-1) j}} & \text { for } j<k \leq i \\ 0 & \text { otherwise }\end{aligned}\right.$

Proof: To prove this, we write out the spatial velocity of frame $\Psi_{k}$ with respect to $\Psi_{(k-1)}$ when $i<k \leq j$ :

$$
\hat{X}_{k} \dot{q}_{k}=\hat{V}_{(k-1) k}^{(k-1)}=\dot{g}_{(k-1) k} g_{(k-1) k}^{-1}=\frac{\partial g_{(k-1) k}}{\partial q_{k}} g_{k(k-1)} \dot{q}_{k}
$$

where $\hat{X}:=\left[\begin{array}{cc}\hat{X}_{\omega} & X_{v} \\ 0 & 0\end{array}\right]$. Comparing these terms we get

$$
\frac{\partial R_{(k-1) k}}{\partial q_{k}}=\hat{X}_{\omega} R_{(k-1) k}, \quad \frac{\partial p_{(k-1) k}}{\partial q_{k}}=\hat{X}_{\omega} p_{(k-1) k}+X_{v} .
$$

We can use this relation in the expression for the partial derivative of $\operatorname{Ad}_{g_{(k-1) k}}$ :

$$
\begin{aligned}
\frac{\partial \operatorname{Ad}_{g_{(k-1) k}}}{\partial q} & =\left[\begin{array}{ccc}
\frac{\partial R_{(k-1) k}}{\partial q_{k}} & \frac{\hat{p}_{(k-1) k}}{\partial q_{k}} R_{(k-1) k}+\hat{p}_{(k-1) k} \frac{\partial R_{(k-1) k}}{\partial q_{k}} \\
0 & \frac{\partial R_{(k-1) k}}{\partial q_{k}}
\end{array}\right] \\
& =\left[\begin{array}{cc}
\hat{X}_{\omega} & \hat{X}_{v} \\
0 & \hat{X}_{\omega}
\end{array}\right]\left[\begin{array}{cc}
R_{(k-1) k} & \hat{p}_{(k-1) k} R_{(k-1) k} \\
0 & R_{(k-1) k}
\end{array}\right] \\
& =\operatorname{ad}_{X_{k}} \operatorname{Ad}_{g_{(k-1) k}}
\end{aligned}
$$

It is now straight forward to show that

$$
\begin{aligned}
\frac{\partial \operatorname{Ad}_{g_{i j}}}{\partial q_{k}} & =\operatorname{Ad}_{g_{i(k-1)}} \frac{\partial \operatorname{Ad}_{g_{(k-1) k}}}{\partial q_{k}} \operatorname{Ad}_{g_{k j}} \\
& =\operatorname{Ad}_{g_{i(k-1)}} \operatorname{ad}_{X_{k}} \operatorname{Ad}_{g_{(k-1) j}} .
\end{aligned}
$$

The proof is similar for $j<k \leq i$.

\section{Implementation We first define the vector}

$$
\xi=(M(q) v)_{V}=\left[\begin{array}{c}
(M(q) v)_{1} \\
\vdots \\
(M(q) v)_{m}
\end{array}\right]=\left[\begin{array}{ll}
M_{V V} & M_{q V}^{\top}
\end{array}\right]\left[\begin{array}{c}
V_{0 b}^{b} \\
\dot{q}
\end{array}\right] .
$$

This gives the adjoint part of the second part of (19) as

$$
\operatorname{ad}_{\xi}=\left[\begin{array}{cccccc}
0 & -\xi_{6} & \xi_{5} & 0 & -\xi_{3} & \xi_{2} \\
\xi_{6} & 0 & -\xi_{4} & \xi_{3} & 0 & -\xi_{1} \\
-\xi_{5} & \xi_{4} & 0 & -\xi_{2} & \xi_{1} & 0 \\
0 & 0 & 0 & 0 & -\xi_{6} & \xi_{5} \\
0 & 0 & 0 & \xi_{6} & 0 & -\xi_{4} \\
0 & 0 & 0 & -\xi_{5} & \xi_{4} & 0
\end{array}\right]
$$

The lower part of the second term in (19) is found from

$$
\frac{\partial^{\top}}{\partial q}(M(q) v)_{V}=\left[\begin{array}{cccc}
\frac{\partial(M v)_{1}}{\partial q_{1}} & \frac{\partial(M v)_{2}}{\partial q_{1}} & \cdots & \frac{\partial(M v)_{6}}{\partial q_{1}} \\
\frac{\partial(M v)_{1}}{\partial q_{2}} & \frac{\partial(M v)_{2}}{\partial q_{2}} & \cdots & \frac{\partial(M v)_{6}}{\partial q_{2}} \\
\vdots & & \ddots & \vdots \\
\frac{\partial(M v)_{1}}{\partial q_{n}} & \frac{\partial(M v)_{2}}{\partial q_{n}} & \cdots & \frac{\partial(M v)_{6}}{\partial q_{n}}
\end{array}\right]
$$

where $\frac{\partial(M v)_{j}}{\partial q_{k}}$ is calculated as

$$
\frac{\partial(M v)_{j}}{\partial q_{k}}=\sum_{i=1}^{6+n} \frac{\partial M_{j i}}{\partial q_{k}} v_{i}
$$

The second part of (18) is computed in the same way. We thus only need to compute the partial derivative $\frac{\partial M(q)}{\partial q_{i}}$ once and use the result in the both in the first and second part of (19).

\section{CONCLUSIONS}

In this paper the dynamic equations of spacecraftmanipulator systems are derived based on Lagrange's equations. The main contribution is to close the gap between manipulator dynamics which are normally derived based on the Lagrangian approach and spacecraft dynamics which are normally derived using other approaches in order to avoid singularities. The proposed framework allows us to derive the dynamics of spacecraft using a minimal, singularity-free representation based on Lagrange's equations which naturally extends to include also the manipulator dynamics. This is derived for the first time in this paper using the proposed framework.

\section{ACKNOWLEDGEMENTS}

P. J. From and J. T. Gravdahl wish to acknowledge the support of the Norwegian Research Council and the TAIL IO project for their continued funding and support for this research. The TAIL IO project is an international cooperative research project led by StatoilHydro and an R\&D consortium consisting of ABB, IBM, Aker Solutions and SKF. During the work with this paper the first author was with the University of California at Berkeley. V. Duindam is sponsored through a Rubicon grant from the Netherlands Organization for Scientific Research (NWO).

\section{REFERENCES}

Bullo, F. and Lewis, A.D. (2004). Geometric Control of Mechanical Systems: Modeling, Analysis, and Design for Simple Mechanical Control Systems. Springer.

Dubowsky, S. and Papadopoulos, E. (1993). The kinematics, dynamics and control of free-flying and free-floating space robotic systems. IEEE Transactions on Robotics and Automation, 9, no. 5.

Duindam, V. (2006). Port-Based Modeling and Control for Efficient Bipedal Walking Robots. Ph.D. thesis, University of Twente. URL http://purl.org/utwente/50829.

Duindam, V. and Stramigioli, S. (2008). Singularityfree dynamic equations of open-chain mechanisms with general holonomic and nonholonomic joints. IEEE Transactions on Robotics, 24(3), 517-526.

From, P.J., Duindam, V., Gravdahl, J.T., and Sastry, S. (2009). Modeling and motion planning for mechanisms on a non-inertial base. International Conference of Robotics and Automation, Kobe, Japan.

Hughes, P.C. (2002). Spacecraft Attitude Dynamics. Dover Publications.

Kozlowski, K. and Herman, P. (2008). Control of robot manipulators in terms of quasi-velocities. Journal of Intelligent and Robotic Systems, 53, no. 3.

Kwatny, H.G. and Blankenship, G. (2000). Nonlinear Control and Analytical Mechanics A Computational Approach. Birkhuser Boston.

Moosavian, S.A.A. and Papadopoulos, E. (2007). Freeflying robots in space: an overview of dynamics modeling, planning and control. Robotica, 25.

Murray, R., Li, Z., and Sastry, S. (1994). A Mathematical Introduction to Robotic Manipulation. CRC Press.

Selig, J.M. (2000). Geometric fundamentals of robotics. Springer. 